\title{
Some results on stability and on characterization of $K$-convexity of set-valued functions
}

\author{
by Tiziana Cardinali (Perugia), Kazimierz Nikodem (Bielsko-Biała) \\ and Francesca Papalini (Perugia)
}

\begin{abstract}
We present a stability theorem of Ulam-Hyers type for $K$-convex set-valued functions, and prove that a set-valued function is $K$-convex if and only if it is $K$-midconvex and $K$-quasiconvex.
\end{abstract}

1. Introduction. In this paper we study two different problems:

(i) stability of the $K$-convexity of a set-valued function;

(ii) characterization of $K$-convex set-valued functions.

The first problem has been studied for functions: in 1941 D. H. Hyers [5] proved that the property of additivity is stable, i.e. if a function $f$ satisfies

$$
|f(x+y)-f(x)-f(y)| \leq \varepsilon,
$$

where $\varepsilon$ is a given positive number, then there exists an additive function $g$ such that

$$
|f(x)-g(x)| \leq \varepsilon .
$$

In 1952 D. H. Hyers and S. M. Ulam [6] stated that the property of convexity is stable, that is, for every function $f: D \rightarrow \mathbb{R}$, where $D$ is a convex subset of $\mathbb{R}^{n}$, satisfying the inequality

$$
f(t x+(1-t) y) \leq t f(x)+(1-t) f(y)+\varepsilon,
$$

for all $x, y \in D, t \in[0,1]$ and some $\varepsilon>0$, there exists a convex function $g: D \rightarrow \mathbb{R}$ and a constant $k_{n}$, depending only on the dimension of the domain, such that

$$
g(x) \leq f(x) \leq g(x)+k_{n} \varepsilon, \quad \forall x \in D .
$$

1991 Mathematics Subject Classification: 26B25, 54C60.

Key words and phrases: set-valued functions, $K$-convex ( $K$-midconvex, $K$-quasiconvex) set-valued functions, Ulam-Hyers stability. 
In 1984 P. W. Cholewa [3] gave a different proof of the theorem of Hyers and Ulam.

Later, in 1988, K. Nikodem $[10]_{1}$ showed that the property of quasiconvexity of a function is also stable.

For the second problem, in $1989 \mathrm{~K}$. Nikodem [10 $]_{2}$ obtained the following characterization for convex functions defined on an open subset of $\mathbb{R}^{n}$ :

$$
f \text { is convex } \Leftrightarrow f \text { is midconvex and quasiconvex. }
$$

Next Z. Kominek [7] and F. A. Behringer [2] showed that (1.5) is also true for functions defined on any convex subset of a real vector space, not necessarily open.

In Section 3 of our note we prove (cf. Theorem 1) that if $D$ is a convex subset of $\mathbb{R}^{n}, K$ a convex cone in $\mathbb{R}^{m}$ and $B$ the closed unit ball of $\mathbb{R}^{m}$, then for every set-valued function $F: D \rightarrow n\left(\mathbb{R}^{m}\right)$ (cf. (2.1)) satisfying

$$
t F(x)+(1-t) F(y) \subset F(t x+(1-t) y)+K+\varepsilon B
$$

for all $x, y \in D, t \in[0,1]$ and some $\varepsilon>0$, there exists a convex set-valued function $G: D \rightarrow n\left(\mathbb{R}^{m}\right)$ such that

$$
F(x) \subset G(x) \subset F(x)+K+j_{n+m} \varepsilon B, \quad \forall x \in D,
$$

where the constant $j_{n+m}$ depends only on the dimension of $\mathbb{R}^{n+m}$.

In Section 4 we prove (cf. Corollary 1 ) that if $D$ is a convex subset of a real vector space, $K$ a closed convex cone of a real topological vector space $Y, t \in(0,1)$ and $F: D \rightarrow C(Y)$ (cf. (2.2)) a set-valued function, then, under some assumption on $Y$ (cf. Remark 1),

$F$ is $K$-convex $\Leftrightarrow F$ is $K$-t-convex and $K$-quasiconvex.

This result contains the mentioned theorems proved in $[10]_{2}$, in $[7]$ and in $[2]$.

Finally, we want to observe that our Theorem 3 is a generalization to set-valued functions of a result of N. Kuhn [8] stating that $t$-convex (singlevalued) functions are midconvex.

2. Let $X$ be a real vector space and $Y$ be a real topological vector space (satisfying the $T_{0}$ separation axiom). For $\alpha, \beta \in \mathbb{R}$ and $S, T \subset Y$ we put $\alpha S+\beta T=\{y \in Y: y=\alpha s+\beta t, s \in S, t \in T\}$. We define

$$
\begin{aligned}
n(Y) & =\{S \subset Y: S \neq \emptyset\}, \\
C(Y) & =\{S \subset Y: S \text { compact, } S \neq \emptyset\}, \\
B C(Y) & =\{S \subset Y: S \text { bounded, convex, } S \neq \emptyset\} .
\end{aligned}
$$


We assume that $D$ is a nonempty convex subset of $X$ and $K$ is a convex cone in $Y$. For fixed $t \in(0,1)$, we say that a set-valued function $F: D \rightarrow$ $n(Y)$ is $K$-t-convex if

$$
t F(x)+(1-t) F(y) \subset F(t x+(1-t) y)+K
$$

for all $x, y \in D$. If $t=1 / 2, F$ is called $K$-midconvex.

We say that $F$ is $K$-quasiconvex if for every convex set $A \subset Y$ the lower inverse image of $A-K$, i.e. the set

$$
F^{-}(A-K)=\{x \in D: F(x) \cap(A-K) \neq \emptyset\},
$$

is convex (cf. $\left.[10]_{3},(2.5)\right)$.

In the case that $Y$ is a normed space, let $B$ be the closed unit ball in $Y$ and $\varepsilon$ a nonnegative number. We say that $F: D \rightarrow n(Y)$ is $\varepsilon$-K-convex if

$$
t F(x)+(1-t) F(y) \subset F(t x+(1-t) y)+K+\varepsilon B
$$

for all $x, y \in D$ and $t \in[0,1]$. We recall that $F$ is $K$-convex if it satisfies (2.4) with $\varepsilon=0$. If $F$ satisfies (2.4) with $\varepsilon=0$ and $K=\{0\}$ it is said to be convex.

$F: D \rightarrow n(Y)$ is said to be weakly K-upper bounded on a set $A \subset D$ iff

(2.5) there exists a bounded set $B \subset Y$ such that $A \subset F^{-}(B-K)$.

Finally, we denote by

$$
\operatorname{Gr} F=\{(x, y) \in X \times Y: x \in D, y \in F(x)\}
$$

the graph of the set-valued function $F$.

3. In this section we present, for $\varepsilon-K$-convex set-valued functions, a theorem analogous to the stability theorem for functions proved by D. H. Hyers and S. M. Ulam in [6] and by P. W. Cholewa in [3].

Using a method similar to Cholewa's [3] we first prove

Lemma 1. Let $X$ be a real vector space, $Y$ a normed space, $D$ a convex subset of $X$ and $K$ a convex cone in $Y$. If a set-valued function $F: D \rightarrow n(Y)$ is $\varepsilon$-K-convex, then for all $p \in \mathbb{N}, x_{0}, \ldots, x_{p} \in D$ and $t_{0}, \ldots, t_{p} \in[0,1]$ with $t_{0}+\ldots+t_{p}=1$, we have

$$
t_{0} F\left(x_{0}\right)+\ldots+t_{p} F\left(x_{p}\right) \subset F\left(t_{0} x_{0}+\ldots+t_{p} x_{p}\right)+K+j_{p} \varepsilon B
$$

where $j_{p}=\min \left\{k_{p}, h_{p}\right\}, k_{p}=\left(p^{2}+3 p\right) /(2 p+2)$, and $h_{p}=m \in \mathbb{N}$ is such that $2^{m-1} \leq p<2^{m}$.

Proof. For $p=1$ the inclusion (3.1) is clear because $j_{1}=k_{1}=h_{1}=1$. Now fix $p>1$ and assume that (3.1) holds for all natural $n<p$. Take $x_{0}, \ldots, x_{p} \in D$ and $t_{0}, \ldots, t_{p} \in[0,1]$ with $t_{0}+\ldots+t_{p}=1$. Without loss 
of generality we may assume that $t_{0} \geq 1 /(p+1)$. Let $t=t_{1}+\ldots+t_{p}$ and $t_{i}^{\prime}=t_{i} / t$ for $i=1, \ldots, p$; then $t \leq p /(p+1)$. Thus

$$
\begin{aligned}
t_{0} F\left(x_{0}\right)+\ldots+ & t_{p} F\left(x_{p}\right)=t_{0} F\left(x_{0}\right)+t\left[t_{1}^{\prime} F\left(x_{1}\right)+\ldots+t_{p}^{\prime} F\left(x_{p}\right)\right] \\
& \subset t_{0} F\left(x_{0}\right)+t\left[F\left(t_{1}^{\prime} x_{1}+\ldots+t_{p}^{\prime} x_{p}\right)+K+k_{p-1} \varepsilon B\right] \\
& \subset F\left(t_{0} x_{0}+\ldots+t_{p} x_{p}\right)+K+\varepsilon B+\frac{p}{p+1} k_{p-1} \varepsilon B \\
& =F\left(t_{0} x_{0}+\ldots+t_{p} x_{p}\right)+K+k_{p} \varepsilon B .
\end{aligned}
$$

Now, let $m \in \mathbb{N}$ be such that $2^{m-1} \leq p<2^{m}$. Put $r=[p / 2]$; then $r<2^{m-1}$ and $p-r-1<2^{m-1}$. Setting $a=t_{0}+\ldots+t_{r}$ and $b=t_{r+1}+\ldots+t_{p}$, we have

$$
\begin{array}{rl}
t_{0} & F\left(x_{0}\right)+\ldots+t_{p} F\left(x_{p}\right) \\
= & a\left[\frac{t_{0}}{a} F\left(x_{0}\right)+\ldots+\frac{t_{r}}{a} F\left(x_{r}\right)\right]+b\left[\frac{t_{r+1}}{b} F\left(x_{r+1}\right)+\ldots+\frac{t_{p}}{b} F\left(x_{p}\right)\right] \\
\subset & a F\left(\frac{t_{0}}{a} x_{0}+\ldots+\frac{t_{r}}{a} x_{r}\right)+b F\left(\frac{t_{r+1}}{b} x_{r+1}+\ldots+\frac{t_{p}}{b} x_{p}\right) \\
& +K+a h_{r} \varepsilon B+b h_{p-r-1} \varepsilon B \\
\subset & F\left(t_{0} x_{0}+\ldots+t_{p} x_{p}\right)+K+\left(1+a h_{r}+b h_{p-r-1}\right) \varepsilon B \\
\subset & F\left(t_{0} x_{0}+\ldots+t_{p} x_{p}\right)+K+[1+a(m-1)+b(m-1)] \varepsilon B \\
= & F\left(t_{0} x_{0}+\ldots+t_{p} x_{p}\right)+K+h_{p} \varepsilon B .
\end{array}
$$

From (3.2) and (3.3) we obtain the assertion.

TheOREm 1. Let $D$ be a convex subset of $\mathbb{R}^{n}$ and $K$ be a convex cone in $\mathbb{R}^{m}$. If a set-valued function $F: D \rightarrow n\left(\mathbb{R}^{m}\right)$ is $\varepsilon-K$-convex, then there exists a convex set-valued function $G: D \rightarrow n\left(\mathbb{R}^{m}\right)$ such that

$$
F(x) \subset G(x) \subset F(x)+K+j_{n+m} \varepsilon B
$$

for all $x \in D$.

Pr o of. Let $W$ be the convex hull of the graph of $F$. We define $G: D \rightarrow$ $n\left(\mathbb{R}^{m}\right)$ by

$$
G(x)=\left\{y \in \mathbb{R}^{m}:(x, y) \in W\right\}, \quad x \in D .
$$

Then $G$ is convex because $\operatorname{Gr} G=W$ is convex. Moreover, $F(x) \subset G(x)$ for all $x \in D$. To prove the second inclusion fix an $x \in D$ and take an arbitrary $y \in G(x)$. Then $(x, y) \in W$. By the Carathéodory Theorem (cf. [12], Theorem 17.1) we have

$$
(x, y)=\sum_{i=0}^{n+m} t_{i}\left(x_{i}, y_{i}\right),
$$


with some $\left(x_{i}, y_{i}\right) \in \operatorname{Gr} F$ and $t_{0}, \ldots, t_{n+m} \in[0,1], t_{0}+\ldots+t_{n+m}=1$. Hence, using Lemma 1, we get

$$
y=\sum_{i=0}^{n+m} t_{i} y_{i} \in \sum_{i=0}^{n+m} t_{i} F\left(x_{i}\right) \subset F(x)+K+j_{n+m} \varepsilon B .
$$

Since this holds for all $y \in G(x)$, the proof is complete.

4. In this section we give two necessary and sufficient conditions for a set-valued function to be $K$-convex. We first need the following lemma which is an analogue of a result obtained for functions by C. T. $\mathrm{Ng}$ and K. Nikodem (cf. [9], Lemma 6).

LEMMA 2. Let $K$ be a closed convex cone in a real topological vector space $Y$. If $F:[0,1] \rightarrow C(Y)$ is $K$-midconvex on $[0,1]$ and $K$-convex on $(0,1)$, then it is K-convex on $[0,1]$.

Proof. Fix $x, y \in[0,1]$ and $t \in(0,1)$, and put $z=t x+(1-t) y$. Let $u=(x+z) / 2$ and $v=(y+z) / 2$. Then $u, v \in(0,1)$ and $z=t u+(1-t) v$. Since $F$ is $K$-convex on $(0,1)$ we get

$$
t F(u)+(1-t) F(v) \subset F(z)+K .
$$

On the other hand, by the $K$-midconvexity of $F$ on $[0,1]$,

$$
\frac{F(x)+F(z)}{2} \subset F(u)+K \text { and } \frac{F(y)+F(z)}{2} \subset F(v)+K .
$$

Therefore, by (4.2) and (4.1),

$$
\begin{aligned}
t F(x)+(1-t) F(y)+F(z) & \subset t(F(x)+F(z))+(1-t)(F(y)+F(z)) \\
& \subset 2 t F(u)+2(1-t) F(v)+K \\
& \subset 2 F(z)+K \subset F(z)+F(z)+K .
\end{aligned}
$$

The set $F(z)+K$ is convex and closed, and $F(z)$ is bounded; so the law of cancellation (cf. [11]) yields the assertion.

THEOREM 2. Let $X$ be a real vector space, $Y$ a real topological vector space, $D$ a convex subset of $X$ and $K$ a closed convex cone in $Y$. Moreover, assume that there exists a family $\left(B_{n}\right)_{n}, B_{n} \in B C(Y)$ (cf. (2.3)), such that

$$
Y=\bigcup_{n \in \mathbb{N}}\left(B_{n}-K\right)
$$

Then a set-valued function $F: D \rightarrow C(Y)$ is $K$-convex if and only if it is K-midconvex and K-quasiconvex. 
P r o of. The necessity is trivial (cf. $[10]_{3}$, Theorem 2.9). Now suppose $F$ is $K$-midconvex and $K$-quasiconvex. Fix $x, y \in D$, and define $H:[0,1] \rightarrow C(Y)$ by

$$
H(t)=F(t x+(1-t) y), \quad \forall t \in[0,1] .
$$

Clearly $H$ is $K$-quasiconvex; therefore for all $n \in \mathbb{N}$, the set

$$
H^{-}\left(B_{n}-K\right)=\left\{t \in[0,1]: H(t) \cap\left(B_{n}-K\right) \neq \emptyset\right\}
$$

is an interval in $\mathbb{R}$. In view of (4.3) we have

$$
\bigcup_{n \in \mathbb{N}} H^{-}\left(B_{n}-K\right)=[0,1],
$$

and so we can find a natural number $p$ such that

$$
\text { int } H^{-}\left(B_{p}-K\right) \neq \emptyset \text {. }
$$

By the $K$-midconvexity of $F$ it follows that $H$ is $K$-midconvex on [0,1], and (cf. (4.5) and (4.6)) $H$ is weakly $K$-upper bounded (cf. (2.5)) on $H^{-}\left(B_{p}-K\right)$, which has nonempty interior; then using Corollary 3.3 of $[10]_{3}$ we deduce that $H$ is $K$-continuous on $(0,1)$. Consequently, $H$ is $K$-convex on $(0,1)$ (cf. $[10]_{3}$, Theorem 3.1 or [1], Theorem 4.2 ) and so it follows by Lemma 2 that $H$ is $K$-convex on $[0,1]$. Therefore, by $(4.4)$,

$$
\begin{aligned}
t F(x)+(1-t) F(y) & =t H(1)+(1-t) H(0) \subset H(t)+K \\
& =F(t x+(1-t) y)+K,
\end{aligned}
$$

which proves the $K$-convexity of $F$.

Remark 1. The assumption (4.3) is trivially satisfied if $Y$ is a normed space. It is also fulfilled if there exists an order unit in $Y$, i.e. an element $e \in Y$ such that for every $y \in Y$ we can find an $n \in \mathbb{N}$ with $y \in n e-K$ (then we can assume $\left.B_{n}=\{n e\}\right)$. In particular, if int $K \neq \emptyset$, then every element of int $K$ is an order unit in $Y$.

THEOREM 3. Let $X$ be a real vector space, $Y$ be a real topological vector space, $D$ a convex subset of $X$ and $K$ a closed convex cone in $Y$. Let $t$ be a fixed number in $(0,1)$. If a set-valued function $F: D \rightarrow C(Y)$ is $K$-t-convex, then it is K-midconvex.

Proof. Observe first that $F(x)+K$ is convex for all $x \in D$ because

$$
t F(x)+(1-t) F(x) \subset F(x)+K
$$

and $F(x)+K$ is closed. 
Let $x, y \in D$; using the $K$-t-convexity of $F$ we get

$$
\begin{aligned}
& t(1-t) F(x)+t(1-t) F(y)+[1-2 t(1-t)] F\left(\frac{x+y}{2}\right) \\
& \subset t\left[(1-t) F(x)+t F\left(\frac{x+y}{2}\right)\right]+(1-t)\left[t F(y)+(1-t) F\left(\frac{x+y}{2}\right)\right] \\
& \subset t F\left((1-t) x+t \frac{x+y}{2}\right)+(1-t) F\left(t y+(1-t) \frac{x+y}{2}\right)+K \\
& \subset F\left(\frac{x+y}{2}\right)+K \\
& \subset 2 t(1-t) F\left(\frac{x+y}{2}\right)+[1-2 t(1-t)] F\left(\frac{x+y}{2}\right)+K .
\end{aligned}
$$

Since the set $2 t(1-t) F\left(\frac{x+y}{2}\right)+K$ is convex and closed and the set $[1-2 t(1-t)] F\left(\frac{x+y}{2}\right)$ is bounded, by the law of cancellation we obtain

$$
t(1-t) F(x)+t(1-t) F(y) \subset 2 t(1-t) F\left(\frac{x+y}{2}\right)+K .
$$

Hence

$$
\frac{1}{2}[F(x)+F(y)] \subset F\left(\frac{x+y}{2}\right)+K,
$$

which was to be proved.

R e mark 2. In the case of real (single-valued) functions the above result is a consequence of the theorem of N. Kuhn [8]. The idea of the presented proof is taken from Lemma 1 of [4].

As an immediate consequence of Theorems 2 and 3 we obtain the following

Corollary 1. Let $X$ be a real vector space, $Y$ a real topological vector space, $D$ a convex subset of $X, K$ a closed convex cone in $Y$ and $t$ a fixed number in $(0,1)$. Moreover, assume that there exists a family $\left(B_{n}\right)_{n}, B_{n} \in$ $B C(Y)$, such that

$$
Y=\bigcup_{n \in \mathbb{N}}\left(B_{n}-K\right) .
$$

Then a set-valued function $F: D \rightarrow C(Y)$ is $K$-convex if and only if it is $K$-t-convex and $K$-quasiconvex.

Remark 3. Observe that, in the case where $K=\{0\}$, it is sufficient to require that the values of the set-valued function in Lemma 2, Theorem 2, Theorem 3 and Corollary 1 are closed and bounded (and not necessarily compact). The corresponding proofs are similar to those given above. 


\section{References}

[1] A. Averna e T. Cardinali, Sui concetti di K-convessità (K-concavità) e di Kconvessità ${ }^{*}$ (K-concavità ${ }^{*}$ ), Riv. Mat. Univ. Parma (4) 16 (1990), 311-330.

[2] F. A. Behringer, Convexity is equivalent to midpoint convexity combined with strict quasiconvexity, Optimization (ed. K.-H. Elster, Ilmenau, Germany), 24 (1992), 219-228.

[3] P. W. Cholewa, Remarks on the stability of functional equations, Aequationes Math. 27 (1984), 76-86.

[4] Z. Daróczy and Z. Páles, Convexity with given infinite weight sequences, Stochastica 11 (1987), 5-12.

[5] D. H. Hyers, On the stability of the linear functional equation, Proc. Nat. Acad. Sci. U.S.A. 27 (1941), 411-416.

[6] D. H. Hyers and S. M. Ulam, Approximately convex functions, Proc. Amer. Math. Soc. 3 (1952), 821-828.

[7] Z. Kominek, A characterization of convex functions in linear spaces, Zeszyty Nauk. Akad. Górniczo-Hutniczej 1277, Opuscula Math. 5 (1989), 71-74.

[8] N. Kuhn, A note on t-convex functions, in: General Inequalities 4 (Proc. Oberwolfach 1983), Internat. Ser. Numer. Math. 71, Birkhäuser, 1984, 269-276.

[9] C. T. Ng and K. Nikodem, On approximately convex functions, Proc. Amer. Math. Soc., to appear.

$[10]_{1}$ K. Nikodem, Approximately quasiconvex functions, C. R. Math. Rep. Acad. Sci. Canada 10 (6) (1988), 291-294.

$[10]_{2}$ _- On some class of midconvex functions, Ann. Polon. Math. 50 (1989), 145-151.

$[10]_{3}-$, K-convex and K-concave set-valued functions, Zeszyty Nauk. Politech. Łódz. 559 (Rozprawy Mat. 114) (1989).

[11] H. Rådström, An embedding theorem for spaces of convex sets, Proc. Amer. Math. Soc. 3 (1952), 165-169.

[12] R. T. Rockafellar, Convex Analysis, Princeton University Press, Princeton, N.J., 1970.

DIPARTIMENTO DI MATEMATICA DELL'UNIVERSITÀ

VIA VANVITELLI 1

06100 PERUGIA, ITALY
DEPARTMENT OF MATHEMATICS TECHNICAL UNIVERSITY

WILLOWA 2

43-309 BIELSKO-BIAłA, POLAND 